\title{
Scale Free Processes in Stellar Cluster Formation
}

\author{
Nate Bastian \\ Astrophysics Research Institute, Liverpool John Moores University, \\ 146 Brownlow Hill, Liverpool L3 5RF, UK \\ email: N.J.Bastian@ljmu.ac.uk
}

\begin{abstract}
We review some of the basic population properties of stellar clusters, as well as how they relate to star-formation more broadly within their host galaxies. Despite the common assertion, the vast majority of stars do not form within stellar clusters. For typical galaxies (including the solar neighbourhood), the fraction of stars forming in clusters is $\sim 10 \%$. There are indications however that this fraction increases as a function of increasing star-formation rate surface density, in agreement with model predictions (based on a turbulent ISM and relatively straight-forward prescriptions of star-formation).
\end{abstract}

Keywords. stellar clusters, star formation

\section{Nearby Star-Forming Regions}

While many cluster related studies begin by asserting that "most stars form in clusters", it has become clear in recent years that this depends crucially on how a cluster is defined. It is true that the vast majority of stars form in a clustered fashion, meaning that few stars form in complete isolation. However, the formation of a gravitationally bound collection of stars appears to be relatively rare, making up just $10 \%$ of star-formation in the Milky Way or nearby galaxies. The solar neighbourhood (within $500 \mathrm{pc}$ ) currently is forming about $25 \%$ of its stars in clusters (e.g., Bressert et al. 2010), however, this is dominated by the Orion Nebula Cluster (ONC), which given the rarity of such massive older clusters in the same volume, suggests that the average fraction is significantly lower.

One of the problems with these estimations is that it is difficult to define a cluster at young ages. Since star-formation appears to follow the hierarchical distribution of the ISM, there is no set physical scale, i.e. a preferred scale, from $0.1-100$ pc (e.g., Elmegreen 2011). Ideally one would want to measure the kinematics of stars within a young star-forming region to work out if they are bound (with and without gas). However, this is impractical and also limited by the lack of knowledge of the 3D spatial distribution of the gas and stars. To get around this, Gieles \& Portegies-Zwart (2011) have defined a parameter, $\Pi$, which is the ratio of the stellar age of the system divided by the crossing time. For bound structures, the crossing time remains largely constant, so $\Pi$ increases with age. For unbound associations, due to their expansion, $\Pi$ decreases with age. While the estimation of the crossing time can be difficult for such young system, $\Pi$ offers a rough estimation of whether a given star-forming region will evolve into a bound structure or will diffuse on rapid timescales into the field.

\section{Extragalactic Star Forming Regions}

While it is more difficult to estimate the fraction of star-formation happening within clusters (or the cluster formation efficiency, CFE, also known as $\Gamma$ - Bastian 2008) in 
extragalactic systems, there have been a number of recent works that have attempted to estimate it, and to compare it to local and global properties of the host galaxy. In order to avoid the complications discussed above, these studies have tended to look at slightly older systems (10 - 50 Myr) where associations have dissolved into the field, but cluster dissolution (due to 2-body relaxation and interaction with the ISM) has not become a dominant effect yet. See Adamo \& Bastian (2016†) for a recent review of these works and their main findings.

While still preliminary, these works have found a relation between $\Gamma$ and the starformation rate surface density $\left(\Sigma_{\mathrm{SFR}}\right)$ and in turn the gas surface density $\left(\Sigma_{\text {gas }}\right)$, in the sense that higher $\Sigma_{\mathrm{SFR}}$ leads to higher $\Gamma$ values (Adamo \& Bastian 2016). This means that starburst galaxies may form a larger fraction of their stars within bound clusters than quiescent galaxies. This is compatible with the idea that globular clusters formed in starburst galaxies at high redshift (see, e.g., Kruijssen 2015) and is consistent with the $T_{L}(U)$ relation discovered in young cluster systems by Larsen \& Richtler (2000) .

Silva-Villa et al. (2013) and Adamo et al. (2015) have extended this type of analysis to within an individual galaxy (M83) and found a clear radial trend of decreasing $\Gamma$ with increasing galactocentric distance. The decrease of $\Gamma$ follows the decreasing $\Sigma_{\text {gas }}$ within this galaxy. Upcoming studies using the LEGUS HST survey of other nearby spiral and dwarf galaxies are necessary to place these initial results on a firm statistical footing.

The observational results obtained so far nicely confirm theoretical predictions on the fraction of star-formation happening in clusters, based of the density distribution of starforming gas within galaxies, where clusters represent the extreme high density tail of a continuous distribution (see Kruijssen 2012 for an in depth discussion).

\section{References}

Adamo, A. \& Bastian, N. 2016, in S. Stahler (ed.), The Birth of Star Clusters, Astrophys. and Space Science Library, Vol. 424 (Springer International Publishing), in press.

Adamo, A., Kruijssen, J. M. D., Bastian, N., Silva-Villa, E., \& Ryon, J. 2015, MNRAS, 452, 246 Bastian, N. 2008, MNRAS, 390, 759

Bressert, E., Bastian, N., Gutermuth, R., et al. 2010, MNRAS, 409, L54

Elmegreen, B. G. 2011, EAS Publications Series, 51, 31

Gieles, M. \& Portegies Zwart, S. F. 2011, MNRAS, 410, L6

Kruijssen, J. M. D. 2012, MNRAS, 426, 3008

Kruijssen, J. M. D. 2015, MNRAS, 454, 1658

Larsen, S. S. \& Richtler, T. 2000, A\& A, 354, 836

Silva-Villa, E., Adamo, A., \& Bastian, N. 2013, MNRAS, 436, L69

$\dagger$ A copy of the chapter can be found at:

http://www.astro.ljmu.ac.uk/ njb/Reviews_files/adamo_bastian_final2.pdf

$\ddagger$ This is an indirect estimation of $\Gamma$, and has some degeneracy with the SFH of the host galaxy. However, it is based on purely observational quantities so does not have any of the associated caveats of deriving cluster ages/masses/extinctions. 